\title{
Operational determination of multi-qubit entanglement classes via tuning of local operations
}

\author{
T. Bastin, ${ }^{1}$ C. Thiel, ${ }^{2}$ J. von Zanthier, ${ }^{2}$ L. Lamata,${ }^{3,4}$ E. Solano, ${ }^{5}$ and G. S. Agarwal ${ }^{6}$ \\ ${ }^{1}$ Institut de Physique Nucléaire, Atomique et de Spectroscopie, Université de Liège, 4000 Liège, Belgium \\ ${ }^{2}$ Institut für Optik, Information und Photonik, Max-Planck Forschungsgruppe, \\ Universität Erlangen-Nürnberg, 91058 Erlangen, Germany \\ ${ }^{3}$ Max-Planck-Institut für Quantenoptik, Hans-Kopfermann-Strasse 1, 85748 Garching, Germany \\ ${ }^{4}$ Instituto de Matemáticas y Física Fundamental, CSIC, Serrano 113-bis, 28006 Madrid, Spain \\ ${ }^{5}$ Departamento de Química Física, Universidad del País Vasco - Euskal Herriko Unibertsitatea, Apdo. 644, 48080 Bilbao, Spain \\ ${ }^{6}$ Department of Physics, Oklahoma State University, Stillwater, OK 74078-3072, USA
}

(Dated: November 8, 2018)

\begin{abstract}
We present a physical setup with which it is possible to produce arbitrary symmetric long-lived multiqubit entangled states in the internal ground levels of photon emitters, including the paradigmatic GHZ and W states. In the case of three emitters, where each tripartite entangled state belongs to one of two well-defined entanglement classes, we prove a one-to-one correspondence between welldefined sets of experimental parameters, i.e., locally tunable polarizer orientations, and multiqubit entanglement classes inside the symmetric subspace.
\end{abstract}

PACS numbers: 42.50.Ex, 03.65.Ud, 03.67.Bg, 42.50.Dv

Entanglement is a distinctive property of quantum physics associated with the nonseparable character of multipartite quantum systems. For the case of two-qubit systems, entanglement is well understood and can be precisely quantified [1]. Apart from the trivial disentangled case, three qubits possess two genuine tripartite inequivalent entanglement classes [2, 3]. Efforts have been done recently towards higher number of qubits $[4,[5,6]$, including an inductive method 7], though so far no comprehensive and scalable classification has been developed. In this letter, we introduce a physical setup consisting of $N$ emitters, incoherently radiating single photons that may be absorbed remotely by detectors equipped with polarizers and producing long-lived multiqubit entangled states among the emitters. We show that it is possible to associate well-defined sets of locally tuned polarizer orientations with multiqubit entanglement classes, allowing their monitoring in an operational manner. Hereby, multipath quantum interferences, associated with qubit permutation symmetry, play a key role in explaining the underlying physics.

We consider a chain of $N$ equally separated single photon emitters, say trapped neutral atoms, trapped ions, quantum dots, or any other equivalent physical system with access to similar behaviour. Each emitter defines a three-level $\Lambda$ system, where $|e\rangle$ denotes the excited state and the two long-lived sublevels, $|+\rangle$ and $|-\rangle$, define a qubit. We assume that the transitions between the excited state and the two lower sublevels have an equal wavenumber and dipole moment, and that they are circularly polarized, $\boldsymbol{\sigma}_{+}$and $\boldsymbol{\sigma}_{-}$, respectively. Figure 1 exemplifies the $N$-emitter case discussed throughout this paper. All emitters are initially excited and we will study the cases in which all spontaneously emitted photons are detected by $N$ detectors located in the far-field re-

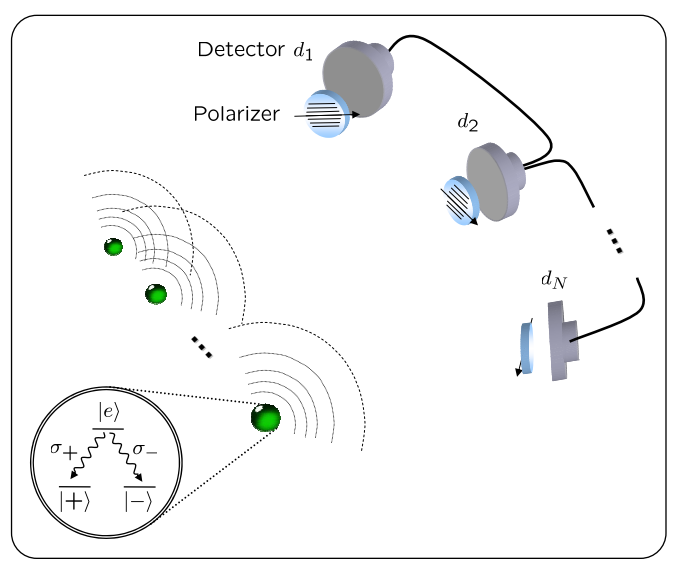

FIG. 1: (color online). Proposed experimental arrangement. $N$ excited emitters are aligned in a row, each of them defining a three-level $\Lambda$-system. A long-lived entangled state is obtained in the $N$ emitter qubits after detecting the $N$ spontaneously emitted photons with $N$ detectors equipped with polarizers. The final $N$-qubit state is tuned and determined by the polarizer orientations.

gion, each of them being equipped with a polarization filter in front. The far-field detection ensures the erasure of which-way information of the arriving photons, and the polarizers allow the generation of quantum superpositions of the lower atomic states when considering arbitrary polarizations. As a consequence each photodetection event projects the emitters onto linear combinations of the long-lived states $|+\rangle$ and $|-\rangle$ [8]. This results at the end in a coherent superposition between the qubit states $| \pm, \ldots, \pm\rangle$. The indeterminacy of which detector 


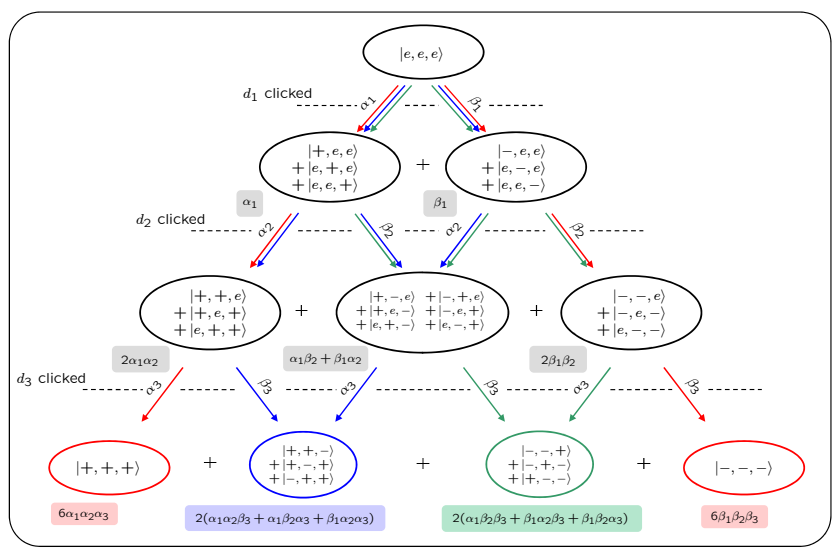

FIG. 2: (color online). Pyramid of entanglement paths for the case of 3 emitters initialized in the excited state $|e, e, e\rangle$. The figure illustrates the intermediate states, horizontally displayed, during three successive photodetection events realized by three detectors $d_{1}, d_{2}$, and $d_{3}$, equipped with general elliptical polarizers oriented along $\boldsymbol{\epsilon}_{1}, \boldsymbol{\epsilon}_{2}$ and $\boldsymbol{\epsilon}_{3}\left(\boldsymbol{\epsilon}_{i} \equiv \alpha_{i} \boldsymbol{\sigma}_{+}+\beta_{i} \boldsymbol{\sigma}_{-}\right)$, respectively. After each detection, the unnormalized global state of the system is the sum of all states in the circles weighed by the tagged prefactors. Leftdown arrows denote $\boldsymbol{\sigma}_{+}$transitions, and right-down arrows represent $\boldsymbol{\sigma}_{-}$ones. The final state components are shown inside colored frames and the colored arrows represent the quantum paths leading to these different components. Only the red circle states are obtained via a single quantum path, while the blue and green ones are the result of three different interfering quantum paths.

has projected which emitter in its ground states implies the existence of many quantum pathways between the initial fully excited atomic state and each of the final state components. This produces a multipath quantum interference effect [9] that we will tune by modifying the polarizer orientations. We remark that several experimental setups may be in condition to implement the concepts introduced in this paper [10, 11, 12, 13].

As will be shown explicitly below, it is always possible to find suitable polarizer orientations to produce any desired state totally symmetric with respect to permutations of the emitters. Hereby, linear polarizers allow the generation of a state of the GHZ type [14], the maximally entangled state

$$
\left|\mathrm{GHZ}_{N}\right\rangle \equiv \frac{1}{\sqrt{2}}\left(|+, \ldots,+\rangle+e^{i \phi}|-, \ldots,-\rangle\right),
$$

with arbitrary relative phase $\phi$, or a separable (product) state

$$
\left|\mathrm{S}_{N}\right\rangle \equiv\left|1_{\phi}, \ldots, 1_{\phi}\right\rangle
$$

with $\left|1_{\phi}\right\rangle \equiv\left(|+\rangle+e^{i \phi}|-\rangle\right) / \sqrt{2}$. An intermediate polarizer configuration permits the generation of the multiqubit state of the W type [2], the weakly entangled state

$$
\left|\mathrm{W}_{N}\right\rangle=\frac{1}{\sqrt{N}}\left(\left|1_{\phi}, 0_{\phi}, \ldots, 0_{\phi}\right\rangle+\ldots+\left|0_{\phi}, \ldots, 0_{\phi}, 1_{\phi}\right\rangle\right),
$$

with $\left|0_{\phi}\right\rangle \equiv\left(|+\rangle-e^{i \phi}|-\rangle\right) / \sqrt{2}$. In the 3 -qubit case, where each tripartite entangled state belongs to one of two welldefined entanglement classes [2, 3]: GHZ or W family, it turns out that even the rotation of a single polarizer allows us to switch from one entanglement class preparation to the other, which are univocally determined by the number of distinct polarizer orientations in the experimental setting. Those polarizer manipulations can be said to be local with respect to the polarizer/detector positions, though they are not local with respect to the qubit positions: it is well known that one cannot convert states from different families into each others using stochastic local operations and classical communication (SLOCC) [15]. Remarkably, the above described local polarization rotations will make possible the transitions

$$
\mathrm{S} \text { class } \leftrightarrow \mathrm{W} \text { class } \leftrightarrow \mathrm{GHZ} \text { class, }
$$

where "S" stems from "separable".

There are several physical systems where the generation of GHZ and W states with three or more qubits have been experimentally achieved: in trapped ions [16, 17], in Rydberg atoms crossing microwave cavities [18], and in photonic systems [19, 20]. Furthermore, other multiqubit entangled states have been realized in different physical setups 21, 22, 23, 24, 25] with different purposes [26] and potential applications in quantum information. Though several paradigmatic entangled states have been produced in the lab, there is no study, to our knowledge, that associates operationally given experimental configurations with multipartite entanglement classes.

The outlined behavior can be understood from the explicit calculation of the different states of the $N$-emitter system after the successive photon detection events. A properly located detector in the far-field with a general elliptical polarizer oriented in the $x y$ plane of the circularly polarized light along the polarization vector $\boldsymbol{\epsilon} \equiv \alpha \boldsymbol{\sigma}_{+}+\beta \boldsymbol{\sigma}_{-}$with arbitrary complex coefficients $\alpha$ and $\beta\left(|\alpha|^{2}+|\beta|^{2}=1\right)$ can implement the operator action [27]

$$
\hat{\mathcal{D}}(\boldsymbol{\epsilon})=\alpha \sum_{j=1}^{N}|+\rangle_{j}\left\langle e\left|+\beta \sum_{j=1}^{N}\right|-\right\rangle_{j}\langle e|,
$$

up to an insignificant prefactor. Here, the sum over $j$ runs over all emitters and $| \pm\rangle_{j}\langle e|$ is the projection operator from state $|e\rangle$ to state $| \pm\rangle$ for emitter $j$. Starting with $N$ emitters in their excited state $|e, \ldots, e\rangle$, the detection of the $N$ emitted photons by $N$ detectors with polarizer configuration $\boldsymbol{\epsilon}_{1}, \ldots, \boldsymbol{\epsilon}_{N}\left(\boldsymbol{\epsilon}_{i} \equiv \alpha_{i} \boldsymbol{\sigma}_{+}+\beta_{i} \boldsymbol{\sigma}_{-}\right)$ projects the emitter system onto the final state $\left|\psi_{f}\right\rangle=$ 


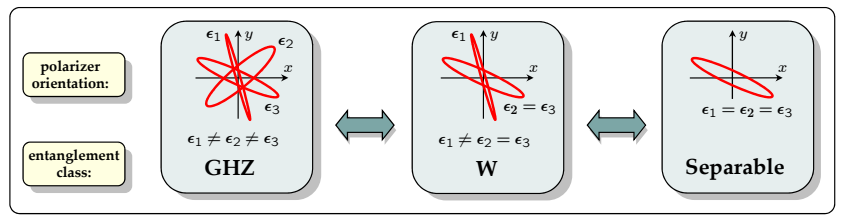

FIG. 3: (color online). Operational monitoring of tripartite entanglement classes by changing the polarization configurations. If the three polarizer orientations are all different, the final state belongs to the GHZ class; if two polarizer orientations are different, the final state belongs to the $\mathrm{W}$ class; if all polarizers are identically oriented, the final state belongs to the $\mathrm{S}$ class.

$\hat{\mathcal{D}}\left(\boldsymbol{\epsilon}_{N}\right) \ldots \hat{\mathcal{D}}\left(\boldsymbol{\epsilon}_{1}\right)|e, \ldots, e\rangle$. Due to the symmetry properties of those operators, the final state as well as all intermediate states are totally symmetric with respect to permutations of the emitters. If entanglement is produced at the end of the detection process, we are ensured that only genuine multipartite entangled states belonging to the accessible symmetric entanglement classes will be generated. The intermediate states produced in the course of the successive photon detection events can be ordered in a pyramidal manner, displaying the various possible quantum paths towards the generation of the desired final state (see Fig. 2 which exemplifies the case $N=3$ ). At the end of the $N$ photon detection process, the system is found in a coherent superposition of all possible product states of the $N$-qubit system in the $| \pm\rangle$ basis. All probability amplitudes related to the different quantum paths add up and yield interference terms (tagged prefactors on Fig. 2). The final state $\left|\psi_{f}\right\rangle$ is found to be a linear combination of all symmetric Dicke states with $k|-\rangle$ excitations [28, 29], $\left|D_{N}(k)\right\rangle$ :

$$
\left|\psi_{f}\right\rangle=\mathcal{N} \sum_{k=0}^{N} c_{k}\left|D_{N}(k)\right\rangle,
$$

where $\mathcal{N}$ is a normalization prefactor, and

$$
c_{k}=\left(C_{N}^{k}\right)^{1 / 2} \sum_{1 \leqslant i_{1} \neq \ldots \neq i_{N} \leqslant N} \beta_{i_{1}} \ldots \beta_{i_{k}} \alpha_{i_{k+1}} \ldots \alpha_{i_{N}},
$$

with $C_{N}^{k}$ the binomial coefficient of $N$ and $k$. Thereby, we can generate any symmetric state with respect to permutations of the emitters using suitable elliptical polarizer orientations. This is a direct application of Vieta's formulas [30] : an arbitrary symmetrical state can be expanded in the Dicke state basis as $\sum_{k}^{N} d_{k}\left|D_{N}(k)\right\rangle$ and is produced in our setup using $K$ polarizers oriented along vectors $\boldsymbol{\epsilon}_{i}$ with $\alpha_{i} / \beta_{i}$ identifying to the $K$ roots of the polynomial $P(z)=\sum_{k}^{N}(-1)^{K-k} \sqrt{C_{N}^{k} / C_{N}^{K}} d_{k} z^{k}$ with $K$ the degree of this polynomial, the remaining polarizers oriented along $\boldsymbol{\sigma}_{+}$.

For example, the maximally entangled state $\left|\mathrm{GHZ}_{N}\right\rangle$ of Eq. (1) is generated with linear polarizers $\boldsymbol{\pi}_{\theta_{k}} \equiv$ $\left(e^{-i \theta_{k}} \boldsymbol{\sigma}_{+}+e^{i \theta_{k}} \boldsymbol{\sigma}_{-}\right) / \sqrt{2}$ oriented along angles

$$
\theta_{k}=\left[\frac{\pi}{2 N}\right]+\frac{\phi}{2 N}+(k-1) \frac{\pi}{N}, \quad k=1, \ldots, N,
$$

or any configuration resulting from permuting the indices. Here, the term inside the square brackets is only present for the case of an even number of emitters. The state $\left|G_{N}\right\rangle$ appears naturally when using all distinct polarizer orientations uniformly shared out over $2 \pi$.

In contrast, we are left with the separable state $\left|\mathrm{S}_{N}\right\rangle$ of Eq. (2) with linear polarizers all identically oriented along the angle $\phi / 2$ :

$$
\theta_{1}=\ldots=\theta_{N}=\frac{\phi}{2} .
$$

The state $\left|\mathrm{W}_{N}\right\rangle$ is also generated with linear polarizers, all except one oriented identically along $\phi / 2$ with the last orthogonal to the $N-1$ first,

$$
\theta_{1}=\ldots=\theta_{N-1}=\frac{\phi}{2}, \quad \theta_{N}=\frac{\phi}{2} \pm \frac{\pi}{2},
$$

or any configuration obtained with permutation of the indices.

For three emitters, those particular results, associating the states $\left|\mathrm{S}_{3}\right\rangle,\left|\mathrm{W}_{3}\right\rangle$, and $\left|\mathrm{GHZ}_{3}\right\rangle$ with certain choices of polarization angles, suggest a wider physical picture. In fact, we can associate in an operational manner specific polarizer configurations of our proposed physical setup with the three paradigmatic entanglement classes appearing in the 3-qubit case : the number of distinct polarizer orientations identifies univocally the entanglement class, be S, W, or GHZ, as shown in Fig. 3.

According to Dür et al. [2], the GHZ class is formed by states characterized by a non vanishing 3 -tangle 31], the $\mathrm{W}$ class by states with a zero 3 -tangle and non-zero single qubit von Neumann entropies, while the separable state class is characterized by zero values of these entanglement measures. In our proposed scheme, the 3-tangle $\tau$ of the final state $\left|\psi_{f}\right\rangle$ reads

$$
\tau=\frac{4}{27} \mathcal{N}^{4}\left|\alpha_{1} \beta_{2}-\alpha_{2} \beta_{1}\right|^{2}\left|\alpha_{1} \beta_{3}-\alpha_{3} \beta_{1}\right|^{2}\left|\alpha_{2} \beta_{3}-\alpha_{3} \beta_{2}\right|^{2}
$$

and vanishes only when 2 polarizers are equally oriented. In this case, the local entropies vanish only when the third polarizer coincides with the two first. When switching from a configuration with three distinct polarizer orientations to a configuration with three identical ones, via the intermediate case where two of them are equal, we transit successively from the GHZ class, to the $\mathrm{W}$ class, and end in the S class, as seen in Fig. 3. This remarkably shows the potentiality of the proposed setup for associating operationally a physical setting with 3-qubit entanglement SLOCC classes of states symmetric with respect to permutations of the emitters. These results encourage a possible generalization to the arbitrary $N$-qubit state. 
Finally, we give an estimation of the expected fidelity of our scheme, e.g., for generating the $\left|\mathrm{GHZ}_{4}\right\rangle$ state using 4 adjacent ions localized in a linear trap. Assuming a $5 \mu \mathrm{m}$ separation between ions with a $5 \mathrm{~nm}$ confinement in the transverse direction and an azimuthal detection window of $1^{\circ}$, we estimate a fidelity of about $90 \%$ for the generation of the 4-qubit state, largely above the $50 \%$ minimal value for proving genuine four-qubit entanglement of the state [32]. With usual ellipsometry techniques, polarizer orientation uncertainties can be made insignificant compared to the finite size effect of the detection window. With an excitation rate of several tens of $\mathrm{MHz}$ [8], the counting rate of the needed fourfold coincident events can be of the order of several tenths of $\mathrm{Hz}$ using CCD cameras covering a fair area in the detection plane. In general, the counting rate decreases with the number of qubits. This might limit the scalability of the scheme as in similar experiments related to entanglement production 8, 13, 19, 25.

Note added.-After submission of this work, we became aware of a nice experimental observation of an entire family of four-photon entangled states [33].

L.L. thanks Alexander von Humboldt Foundation for the funding through a Humboldt Research Fellowship. G.S.A. thanks NSF grant NSF-CCF-0524673 for supporting this collaboration. C.T. and J.v.Z. gratefully acknowledge financial support by the Staedtler foundation. E.S. thanks to Ikerbasque Foundation, EU EuroSQIP project, and UPV-EHU Grant GIU07/40. T.B. thanks F. Bastin, P. Mathonet, P. Lecomte and M. Rigo from University of Liège for helpful discussions.

[1] W. K. Wootters, Phys. Rev. Lett. 80, 2245 (1998).

[2] W. Dür, G. Vidal, and J. I. Cirac, Phys. Rev. A 62, 062314 (2000).

[3] A. Acín, D. Bruß, M. Lewenstein, and A. Sanpera, Phys. Rev. Lett. 87, 040401 (2001).

[4] F. Verstraete, J. Dehaene, B. De Moor, and H. Verschelde, Phys. Rev. A 65, 052112 (2002).

[5] L. Lamata, J. León, D. Salgado, and E. Solano, Phys. Rev. A 75, 022318 (2007).

[6] L. Chen and Y.-X. Chen, Phys. Rev. A 74, 062310 (2006).

[7] L. Lamata, J. León, D. Salgado, and E. Solano, Phys. Rev. A 74, 052336 (2006).

[8] B. B. Blinov, D. L. Moehring, L.-M. Duan, and C. Monroe, Nature 428, 153 (2004).

[9] C. Cabrillo et al., Phys. Rev. A 59, 1025 (1999); X.-L. Feng et al., Phys. Rev. Lett. 90, 217902 (2003); C.-S. Yu et al., Phys. Rev. A 75, 044301 (2007).

[10] U. Eichmann et al., Phys. Rev. Lett. 70, 2359 (1993).

[11] Y. Miroshnychenko et al., Nature 442, 151 (2006).

[12] J. Beugnon, M. P. A. Jones, J. Dingjan, B. Darquié, G. Messin, A. Browaeys, and P. Grangier, Nature 440, 779
(2006).

[13] D. L. Moehring, P. Maunz, S. Olmschenk, K. C. Younge, D. N. Matsukevich, L.-M. Duan, and C. Monroe, Nature 449, 68 (2007).

[14] D. M. Greenberger, M. Horne, A. Shimony, and A. Zeilinger, Am. J. Phys. 58, 1131 (1990).

[15] Only approximated conversions can be realized. See e. g. P. Walther, K. J. Resch, and A. Zeilinger, Phys. Rev. Lett. 94, 240501 (2005).

[16] D. Leibfried et al., Nature 438, 639 (2005).

[17] H. Häffner et al., Nature 438, 643 (2005).

[18] A. Rauschenbeutel, G. Nogues, S. Osnaghi, P. Bertet, M. Brune, J.-M. Raymond, and S. Haroche, Science 288, 2024 (2000).

[19] J.-W. Pan, D. Bouwmeester, M. Daniell, H. Weinfurter, and A. Zeilinger, Nature 403, 515 (2000).

[20] B. P. Lanyon and N. K. Langford, quant-ph/0802.3161 (2008).

[21] J.-W. Pan, M. Daniell, S. Gasparoni, G. Weihs, and A. Zeilinger, Phys. Rev. Lett. 86, 4435 (2001).

[22] Z. Zhao, Y.-A. Chen, A.-N. Zhang, T. Yang, H. J. Briegel, and J.-W. Pan, Nature 430, 54 (2004).

[23] C. A. Sackett et al., Nature 404, 256 (2000).

[24] D. Leibfried, M. D. Barrett, T. Schätz, J. Britton, J. Chiaverini, W. M. Itano, J. D. Jost, C. Langer, and D. J. Wineland, Science 304, 1476 (2004).

[25] N. Kiesel, C. Schmid, G. Tóth, E. Solano, and H. Weinfurter, Phys. Rev. Lett. 98, 063604 (2007).

[26] P. Kok, H. Lee, and J. P. Dowling, Phys. Rev. A 65, 052104 (2002).

[27] G. S. Agarwal, J. von Zanthier, C. Skornia, and H. Walther, Phys. Rev. A 65, 053826 (2002).

[28] In an $N$ spin- $\frac{1}{2}$ system, the Dicke states, usually denoted by $|S, m\rangle$, are defined as the simultaneous eigenstates of both the square of the total spin operator $\hat{\mathbf{S}}^{2}$ and its $z$-component $\hat{S}_{z}$, where $S(S+1) \hbar^{2}$ and $m \hbar$ are the corresponding eigenvalues. The $N+1$ states with the highest value $S=N / 2$ form a special subset of Dicke states that are symmetric under qubit permutation, and can be characterized by the number of excitations $k$ and denoted $\left|D_{N}(k)\right\rangle$. For details see R. Dicke, Phys. Rev. 93, 99 (1954).

[29] C. Thiel, J. von Zanthier, T. Bastin, E. Solano, and G. S. Agarwal, Phys. Rev. Lett. 99, 193602 (2007).

[30] Vieta's formulas are sum-of-roots and productof-roots identities for polynomial. See e. g. E. W. Weisstein, "Vieta's Formulas." From MathWorld - A Wolfram Web Resource. http://mathworld.wolfram.com/VietasFormulas.html

[31] The 3-tangle is a measure of genuine tripartite entanglement that is invariant under permutations. For a formal definition see V. Coffman, J. Kundu, and W. K. Wootters, Phys. Rev. A 61, 052306 (2000).

[32] M. Seevinck and J. Uffink, Phys. Rev. A 65, 012107 (2001).

[33] W. Wieczorek, C. Schmid, N. Kiesel, R. Pohlner, O. Gühne, and H. Weinfurter, Phys. Rev. Lett. 101, 010503 (2008). 